\title{
$\begin{array}{ll}\text { Research Square } & \text { Preprints are preliminary reports that have not undergone peer review. } \\ \text { They should not be considered conclusive, used to inform clinical practice, }\end{array}$ or referenced by the media as validated information. \\ The Effect of lodine-131 Beta-particles in Combination With A-966492 and Topotecan on Radio-sensitization of Glioblastoma
}

Fereshteh Koosha ( $\square$ frshtkoosha@yahoo.com )

Shahid Beheshti University of Medical Sciences School of Paramedical Sciences

\section{Samira Eynali}

Iran University of Medical Sciences

Nazila Eyvazzadeh

Aja University of Medical Sciences Faculty of Paramedicine

\section{Research Article}

Keywords: Radio-sensitization, Targeting therapy, lodine131, Glioblastoma cells, Topotecan

Posted Date: February 25th, 2021

DOI: https://doi.org/10.21203/rs.3.rs-239278/v1

License: (c) (i) This work is licensed under a Creative Commons Attribution 4.0 International License.

Read Full License 
The effect of iodine-131 beta-particles in combination with A-966492 and Topotecan on radio-sensitization of glioblastoma

\section{Fereshteh Koosha $^{1 *}$, Samira Eynali ${ }^{2}$, Nazila Eyvazzadeh ${ }^{3}$}

${ }^{1}$ Department of Radiology Technology, Faculty of Allied Medical Sciences, Shahid Beheshti University of Medical Sciences, Tehran, Iran

${ }^{2}$ Radiation Biology Research Center, Iran University of Medical Sciences, Tehran, Iran

${ }^{3}$ Radiation Sciences Research Center, Faculty of Para medicine, AJA University of Medical Sciences, Tehran, Iran

* Corresponding author: Fereshteh Koosha, Ph.D,

Assistant Professor, Department of Radiology Technology, Faculty of Allied Medical Sciences, Shahid Beheshti University of Medical Sciences, Darband St, Ghods Sq., Tehran, Iran .

Tel: +98-2122717503

ORCID: 0000-0001-9190-3157

E-mail: f.koosha@sbmu.ac.ir,frshtkoosha@yahoo.com 


\begin{abstract}
Glioblastoma tumors are resistant to radiotherapy, and the need for drugs to induce radiosensitization in tumor cells has always been a challenge. Besides, radiotherapy using targeted radionuclide is effective even for resistant tumors. Poly (ADP-ribose) polymerase (PARP) and topoisomerase I enzymes have critical roles in the repairmen of DNA damage in cells. Thus, the inhibition of the activity of these enzymes can prevent the process of DNA repair and lead to the accumulation of damaged DNA in cells, resulting in the induction of cell death in tumors. In the current study, we investigated the effect of beta-particles of iodine-131 in combination with Topotecan (TPT), as the inhibitor of topoisomerase I, and A-966492, as the inhibitor of the PARP enzyme to increase radio-sensitivity of glioblastoma cells.

The U87MG cell line (a human glioblastoma cell line) were cultured in Poly-Hema-coated flasks to reach $300 \mu \mathrm{m}$-diameter spheroids. Then, the cells were treated with non-toxic concentrations of A-966492 and TPT. The viability of the cells treated with iodine131 in combination with A-966492 and TPT was determined by the clonogenic assay. The expression level of the gamma-H2AX protein, as a biomarker of DNA double-strand breaks, was measured by the immunofluorescence staining method to examine the impact of A-966492 $(1 \mu \mathrm{M})$, TPT, and radiation on the induction cell death.
\end{abstract}

The combination of A-966492 and TPT with radiation resulted in the enhanced cell death, and sensitizer enhancement ratios at 50\% survival (SER50) were 1.25 and 1.45, respectively. Radioand chemo-sensitization were promoted when iodine-131 was combined with A-966492 and TPT, with the SER50 of 1.68. Also, the expression of $\gamma$-H2AX was significantly increased in cells treated with A-966492 and TPT combined with radiation.

The results demonstrated that iodine-131, in combination with A-966492 and TPT, had marked effects on radio-sensitizing and can be used as a targeted radionuclide for targeting radiotherapy in combination with PARP and topoisomerase I inhibitors to improve radiotherapy in clinics.

Keywords: Radio-sensitization; Targeting therapy; Iodine131; Glioblastoma cells; Topotecan 


\section{Introduction}

Although multidisciplinary methods and different therapeutic modalities have been used for the treatment of glioblastoma, patients with glioblastoma deal with poor prognosis [1]. Aggressive surgery, chemotherapy, and radiotherapy are common therapeutic options used for the treatment of patients with glioblastoma. The 5-year survival for glioblastoma has been reported to approximately $3 \%$, while the 2-year survival is approximately $8.2 \%$ (i4). Radiotherapy has been used for the cure of high- and low-grade gliomas for many decades; however, the delivery of lowor high-dose radiation to tumors leads to ineffective treatment or the emergence of adverse effects on normal tissues, respectively[2, 3]. The resistance of glioma tumors to current treatment modalities urges scientists to investigate the efficacy of novel and nontoxic radio-sensitizers in order to achieve a desired therapeutic response. Apparently, late radiation toxicity is irreversible, and its occurrence may be increased with the dose of radiation $[4,5]$. Hence, the application of radiation in combination with nontoxic doses of Radiosensitizers could deliver effective therapeutic doses to tumor cells and lead to lower side effects on the organ at risk and finally result in the successful treatment of glioma. It is now known that DNA is the most significant target for the biological effects of ionizing radiation [6]. The interaction of ionizing radiation with DNA leads to multiple damages to DNA, namely single-strand break (SSB) and double-strand break (DSB). The induction of SSB by megavoltage X-ray is not cytotoxic [7]. Poly (ADP) ribose polymerase proteins, such as (PARP1) and (PARP2), are considered the key enzymes in repairing SSB, rapidly binding to SSB, and activate the repair process in cells [8]. Hence, the inhibition of PARP1 and PARP2 may leave SSB unrepaired and induce DSB, subsequently leading to radiosensitization and cell death. A-966492, $\mathrm{C}_{18} \mathrm{H}_{17} \mathrm{FN} 4 \mathrm{O}$, is a dual inhibitor of PARP1 and PARP2 enzymes, which is orally bioavailable and can cross the blood-brain barrier $[9,10]$. 
Topotecan (TPT) is derived from Camptothecin and acts as the inhibitor of topoisomerase I. Following the formation of SSB, topoisomerase I covalently binds to the 3 '-end of DNA and form the Topo I-DNA cleavable complex. Topo I inhibitors, such as TPT, are able to stabilize this complex and extend the lifetime of DNA strand break; so, the repair process cannot successfully accomplish, and the persistence of DSB leads to cell death [11]. In addition to the application of radio-sensitizers, targeting radiotherapy is the selective delivery method to convey the beams to cancer cells in a way that causes minimal toxicity on surrounding normal tissues [12]. Iodine-131, with a physical half-life of 8.04 days, can be used as a radiopharmaceutical agent, and its irradiation consists of beta $(606 \mathrm{keV})$ and gamma $(364 \mathrm{keV})$ particles. Beta-particles are capable of inducing cell damage and could be easily labeled with Tositumomab or MIBG [13]. Iodine-131, as a low-cost and easy labeling radionuclide, in addition to the creation of self-dose in surrounding cells, is able to transfer cross-dose to adjacent and distant cells, creating DNA strand breaks in cells and induce apoptosis. Therefore, in the case of receiving low levels of self-dose by cancer cells, the cross-dose can compensate for such deficiency and deliver higher doses of radiation to tumor cells. Previously, iodine-131 was used for the treatment of neuro and glioblastoma $[14,15]$. Therefore, the current study aimed to examine the effects of A-966492, as a PARP1 inhibitor, and TPT, as a Topoisomerase I inhibitor, in combination with radiotherapy on glioblastoma cells. The complexity, heterogeneity, plasticity, and diversity of the human tumor environment cannot be simulated in 2D cell culture models; thus, spheroids, as a 3D cell culture model, was used to simulate the in-vivo conditions. 


\section{Materials and methods}

\subsection{Cell line}

At first, the U87MG cell line, derived from the human glioblastoma multi-form cells, was procured from the Pasteur Institute, Tehran, Iran. U87MG cells were cultured in cell-culture flasks, containing DMEM (Dulbecco's modified Eagle's medium) supplemented with penicillin, streptomycin (GmbH/PAA, Austria), and 10\% fetal bovine serum (FBS; Gibco/Invitrogen, USA) at $37^{\circ} \mathrm{C}$ in a $5 \% \mathrm{CO} 2$ and $95 \%$ air atmosphere. When the morphology of the cells became spheroids, they were cultured based on the liquid overlay technique. In this technique, T25-flasks were coated with Poly-HEMA (Sigma/Aldrich, Germany) [15]. Afterward, a total of $5 \times 10^{5}$ cells were seeded onto a pre-coated T25-flask containing $5 \mathrm{ml}$ of DMEM supplemented with $10 \%$ FBS. The T25-flask was incubated at $37^{\circ} \mathrm{C}$ in a 5\% $\mathrm{CO} 2$ and $95 \%$ air atmosphere for 19 days [16] to reach a diameter of $300 \mu \mathrm{m}$. A fresh cell culture medium was replaced with the half of the cultured medium twice a week.

\subsection{Treatment and irradiation procedures}

When spheroids reached a diameter of $300 \mu \mathrm{m}$, they were treated with A-966492, TPT, and a combination of A-966492 and TPT. Glioblastoma spheroids were treated with $1 \mu \mathrm{M} \mathrm{A}-966492$ for 1 hour prior to irradiation at various doses of 1,1.5, and 2Gy of iodine-131. Cells irradiated with iodine-131 were incubated with a solution containing 10 mci iodine- 131 dissolved in $0.2 \mathrm{M} \mathrm{NaOH}$ in different time intervals to be exposed to the above-mentioned doses determined based on previous studies [16, 17]. Lastly, the cells were treated with TPT for 2 hours. 


\subsection{Colony-formation assay}

In this assay, cells treated with TPT and various doses of irradiation were seeded onto 60 mm-dishes at various densities. Then, the cells were cultured in DMEM supplemented with 10\% FBS. About two weeks later, colonies formed in dishes were rinsed with phosphate-buffered saline (PBS), fixed with 5\% formaldehyde, and finally stained with crystal violet. The colony was defined by a cell population of at least 50 cells. The index of surviving fraction was obtained by dividing the number of colonies of treated cells by the control cells. The survival curve of irradiation was fitted to the linear-quadratic equation. The surviving fraction is measured by the below formula:

surviving fraction $=\exp \left(-\alpha \mathrm{D}-\beta \mathrm{D}^{2}\right)$, where $\mathrm{D}$ is the radiation dose.

The sensitizer enhancement ratio (SER) was applied to assess the interaction of the drugs with radiation. The SER value is calculated by fitting it into the Linear-Quadratic (LQ) model according to the following formula: $\mathrm{SER}_{\mathrm{x}} \%=\frac{d x \%(\text { no drug })}{d x \%(\text { drug })}$

where $\mathrm{dx} \%$ (no drug) is the radiation dose (Gy) needed to cause $\mathrm{x} \%$ cell survival in the absence of the drug, while dx\% (drug) is the radiation dose (Gy) in the presence of the drug (e.g., TPT or A-966492). The values of SER were determined at doses related $10 \%$ and $50 \%$ surviving fractions.

\subsection{Immunocytochemical analysis}

Following the treatment of cells with the two dugs mentioned earlier and their exposure to irradiation earlier, spheroids were rinsed with $1 \mathrm{ml}$ of PBS, followed by the addition of $2 \mathrm{~N} \mathrm{HCL}$ and incubated for 20 minutes before being washed with borate buffer. Then, the formed spheroids were permeabilized with goat serum and Triton X-100 for 45 minutes. The spheroid were treated with the primary antibody against the gamma-H2A.X phosphor-S139 antibody (dilution 1:1000, 
Abcam, UK) and kept at $4^{\circ} \mathrm{C}$ overnight. Afterward, the cells were treated with $100 \mu 1$ of the FITCconjugated secondary antibody and incubated at $37^{\circ} \mathrm{C}$ for 2 hours in the dark. After that, the cells were washed with PBS to remove unconjugated antibodies; then transferred to glass slides, and their nuclei were stained with propidium iodide (PI). Then, the fluorescence intensity of labelled cells were analyzed by fluorescence microscopy (Nikon, Japan). The green-colored signals of the cells stained with phosphor-H2AX antibodies were semi-quantified by the Image-J software.

\subsection{Western blot}

In this technique, cells were collected and lysed in a lysis buffer solution (RIPA, Beyotime Institute of Biotechnology), containing the protease inhibitors (PMSF, Aladdin). The equal amounts of extracted proteins $(40 \mu \mathrm{g})$ were subjected to electrophoresis and separated under non-reducing conditions on 5-12\% Tris-glycine polyacrylamide gel. Antibodies against Bcl-2, Bax, p53 (Abcam, Cambridge, US), and $\beta$-actin (Santa Cruz, US) were diluted at a ratio of 1:1000. Secondary antibodies used to track the primary antibodies were HRP-conjugated goat anti-rabbit IgGs (Santa Cruz, US) or HRP-conjugated goat anti-mouse IGs (Santa Cruz, US). The secondary antibodies were visualized by the enhanced chemiluminescence according to the manufacturer's instructions (Amersham Life Sciences Inc., Arlington Heights, IL). The obtained results were assessed by the densitometry analysis using the ImageJ software. The $\beta$-actin protein was employed as the internal control.

\subsection{Statistical analysis}

The statistical analysis was conducted by the SPSS software version 16 and the differences between the experimental groups were evaluated by independent T-test or one-way analysis of variance (ANOVA) followed by Schaffer's post hoc test. The statistical level of significance was set as $p<0.05$. 


\section{Results}

\subsection{The impact of therapeutic agent in combination with iodine-131 on colony formation}

The results of the clonogenic assay following the combinatory treatment with A-966492, TPT, and iodine-131 on $300 \mu \mathrm{m}$-glioblastoma spheroids are shown in Figure 1. According to Figure 1 A\& B), the survival fraction was significantly decreased in a dose-dependent manner in cells treated with each of TPT and A-966492, indicating the improved radio-sensitivity in U87MG spheroids even at low doses. Also, Figure 1D demonstrates that the survival fraction was significantly lower in cells treated with A-966492 compared with those treated with TPT, suggesting that A-966492 had highest radio-sensitizing effects. Also, the combination of TPT, A-966492, and iodine131 significantly $(\mathrm{p}<0.05)$ reduced the surviving fraction in comparison with the cells exposed to radiation alone. Table 1 shows the $\alpha$ and $\beta$ values in the linear-quadratic equation, which are used for calculating the surviving fraction. The SER50 values were calculated for cells treated with TPT, A-966492, and the combination of both in the presence of iodine-131. The combination of iodine131 and A-966492 led to substantial radio-sensitizing effects with the SER50 value of 1.25, which is significantly higher than that of cells treated with TPT and radiation (1.45). This confirms that A-966492 is an effective radio-sensitizer when used for the treatment of the U87MG cell-line. The combination of radiation, TPT, and A-966492 had the strongest effects compared to any single usage of each with the SER50 value of 1.68. It seems A-966492 increases the sensitivity of the cells to both radiation and TPT.

\subsection{Determining gamma-H2AX formation after irradiation and drug treatment}

After the treatment of $300 \mu \mathrm{m}$-spheroids with TPT and A-966492 at the presence of iodine-131, the induction of DSB was detected by analyzing the expression of the $\gamma-\mathrm{H} 2 \mathrm{AX}$ protein in U87MG 
cells using anti-gamma-H2A.X phosphoS139 antibody (Abcam, UK). In this experiment, cells were fixed immediately after drug treatment and irradiation. The level of gamma-H2AX expression, shown in Figure 2, was calculated by the ImageJ software, denoting the formation of DSB. Figure 3 shows that in cells treated with radiation alone, the expression of gamma-H2AX was significantly higher than the control cells. Figure 3 A\&B shows that in cells treated with A966492, gamma-H2AX expression was significantly higher than the control cells, while the difference in the expression levels of gamma-H2AX between the TPT-treated and control cells was not statistically significant, implying that A-966492 can specifically induce DSB. There was a significant difference in the number of gamma-H2AX-positive cells that were treated with A966492 and iodine-131 or TPT and iodine-131, confirming that both drugs individually have the capability to enhance radio-sensitization at doses of $1,1.5$, and $2 \mathrm{~Gy}$. According to Figure 3C, the level of gamma-H2AX expression in cells treated with both A-966492 and TPT combined with doses of 1, 1.5, 2 (Gy) of iodine-131 was significantly $(\mathrm{p}<0.05)$ higher than cells treated with iodine-131 alone, suggesting that combinatory treatment with A-966492 and TPT is able to enhance the induction of DSB in the presence of iodine-131 irradiation.

\subsection{Effect of A-966492 and TPT and iodine-131 irradiation on the expression levels of Bcl-2, Bax, p53, and gamma-H2AX proteins}

In order to reveal the mechanisms underlying the apoptotic role of A-966492 and/or in combination with iodine-131, the levels of apoptosis-related proteins, such as Bcl-2, Bax, and p53 were assessed by the western blot analysis in control cells and those treated with 1.5 (Gy), 2 (Gy), A-966492+1 (Gy), A-966492+1.5(Gy), TPT+1 (Gy), TPT+1.5(Gy), A-966492+ TPT+1 (Gy). The

results demonstrated that the expression rates of $\mathrm{Bax} / \mathrm{Bcl}-2$ and $\mathrm{p} 53$ showed the maximum 
expression levels in cells irradiated by 2 Gy of iodine-131 compared with other treated groups. The difference in the expression rate of Bax/Bcl-2 and p53 was not statistically (p>0.05) significant in cells treated with A-966492+ TPT+1 (Gy). Also, the increase in the expression of Bax, along with the reduction in the expression of Bcl-2, was remarkably $(\mathrm{p}<0.05)$ higher in cells treated with the combination of drugs compared to those treated with A-966492 or TPT alone. As shown in Figure 3, the expression level of gamma-H2AX was more pronounced in cells treated with the two drugs and iodine-131, indicating that the induction of DSB in spheroids is in line with our immunocytochemical results. The results indicated that the expression of the gamma-H2AX protein was significantly $(\mathrm{p}<0.05)$ higher in cells treated with the combination of A-966492 and TPT compared with those treated with A-966492 or TPT alone.

\section{Discussion}

Surgery, chemotherapy, and radiotherapy are conventional methods for the treatment of glioblastoma. The adverse reactions occurring in the tumor microenvironment, as well as the resistance of tumor cells to radiation, have limited the efficacy of radiotherapy. Targeted radionuclide therapy is considered one of the most currently developing directions of nuclear medicine. This therapeutic method has fewer side effects on normal tissues and allows drug delivery to tumor tissue in a targeted manner, resulting in the development of systemic therapy of cancer. The delivery of high doses of radiation to cancer cells and exemption of organ at risks are considered the primary purpose in radiotherapy [18]. The ionizing radiation, thereby the induction

of SSB and DSB in DNA of the cells, is capable of inhibiting tumor cell replication and inducing cell death. Some damages caused by ionizing radiation may be repairable by the cell repair process, which is activated in response to the expression of several proteins. Any deficiencies in the cell repair process may lead to the development of irreversible injuries and apoptosis [19]. 
Correspondingly, the inhibitors of PARP and topoisomerase I enzymes have been extensively investigated to sensitize cancer cells to radiation. As shown in our previous study, the combination usage of A-966492, as a PARP1 inhibitor, and TPT, as a topoisomerase I inhibitor, led to the sensitization of glioblastoma spheroids in the presence of X-ray [20]. In the current study, with the emphasis on targeting approaches to cure glioblastoma tumors, A-966492 and TPT were used in combination with beta-particles of iodine-131 to induce cell death in glioblastoma spheroids. Our study showed that the surviving fraction of glioblastoma spheroids treated with each of the two drugs and iodine-131 was lower than cells irradiated with different doses of beta-particles of iodine-131 alone. Interestingly, the reduction in survival fraction was more pronounced in cells treated with A-966492+ iodine-131 in comparison with those treated with TPT+ iodine-131 with the SER50 values of 1.45 and 1.34, respectively. This result may be due to further amplification of DNA repair pathways and activation of PARP proteins. A-966492 promotes radio-sensitivity by inhibition of the repair process when SSB occurs as well as the induction of DSB, leading to radio-sensitization caused by the collapse of the replication forks. PARP inhibitors have been extensively studied in-vitro and in-vivo on different tumor cell lines and caused radio-sensitivity enhancement by 1.3-2 folds. Also, according to Table 1, the strongest radio-sensitizing effect with the SER50 value of 1.68 was detected in cells treated with the combination of iodine-131, TPT, and A-966492, implying the synergic effects of the two drugs and irradiation. Immediately following the formation of DSB in response to irradiation or chemotherapeutic agents, phosphorylation of $\mathrm{H} 2 \mathrm{AX}$ (gamma-H2AX) occurred at the site of DSB and was evident after antibody labeling. The phosphorylation of H2AX at the site of DSB is induced by irradiation and radio-sensitizers, showing that the expression of gamma- $\mathrm{H} 2 \mathrm{AX}$ was more pronounced in spheroids treated with A-966492 alone and also in cells treated with the combination A-966492, TPT, and 
iodine-131. This evidence indicates that A-966492 is a potent radio-sensitizer in the induction of DSB in glioblastoma cells, as confirmed with the western blot analysis. The results showed that the expression levels of Bax and p53 in cells treated with 2 Gy of iodine-131 had no significant difference with cells treated with A-966492, TPT, and 1 Gy of iodine-131. It can be concluded that the cytotoxic effect of iodine-131 in higher doses is alone equal to the combination use of radio-sensitizers with lower doses of iodine-131. It seems that apoptosis could be intensified with lower doses of irradiation in glioblastoma cells in the presence of radio-sensitizers (A-966492, TPT).

On the other hand, in order to reach a high therapeutic index, targeted radionuclide therapy can be used to deliver high doses of radio-sensitizers to tumors. The effectiveness of TPT has been shown in the induction of DSB in different types of cancer, including breast, ovaries, lung, leukemia, nonHodgkin lymphoma, melanoma, colorectal carcinoma, and glioblastoma[13]. Also, with regard to other investigations, 131I-Metaiodobenzylguanidine (131I-MIBG) has been demonstrated to have beneficial effects on the treatment of pheochromocytoma, paraganglioma [21, 22], medullary thyroid cancer [23], and carcinoid tumors [24, 25] with a high efficiency ranging from 30\%-75\%.

In a study conducted by Neshastehriz and colleagues, they revealed that iodine-131 is able to cause higher degrees of DSB in comparison with $6 \mathrm{MV}$ X-ray when used at the same dose as a result of its crossfire effect and spatial distribution of energy in various angles. Besides, they indicated that the combinatory use of chemotherapy and iodine-131 exhibited higher efficacy compared with radiotherapy with a dose of $6 \mathrm{MV}$ X-ray against glioblastoma cells [13]. By means of the crossfire effect, iodine-131 emits lethal doses of irradiation, preventing the proliferation of adjacent tumor cells [26]. The tumor size has an essential role in the crossfire effect. In micro-metastasis, where the diameter of tumors is less than the size of particles, a part of beta-particle energy is dispersed 
out of tumors. Thus, iodine-131 is beneficial for the treatment of tumors and advanced micrometastatic cells that their diameter is more than the range of beta particles [27]. In one study, patients with recurrent glioma received a single dose of TM-601 radiolabeled with 10 mci iodine-

131. ${ }^{131}$ I-TM-601 was administered intraperitoneally and exhibited marked anti-cancer effects[14].

Therefore, our observation is in agreement with the evidence indicating that irradiation of glioblastoma cells with beta-particles of iodine-131 in combination with radio-sensitizers substantially reduced the survival fraction and improved the radiotherapy response in-vitro. Taken together, it appears that irradiation with beta particles of iodine-131 along with TPT and A-966492 may be an effective approach in radiotherapy of glioblastoma tumors.

\section{Ethics approval and consent to participate}

Not applicable.

\section{Consent for publication}

Not applicable.

\section{Availability of data and materials}

Data and material are available.

\section{Competing interests}

The authors declare no conflict of interest.

\section{Funding}


No funding was received.

\section{Authors' contributions}

F.K. and N.E. designed the study and supervised the project. F.K. and S.E. carried out the experiments, analyzed data and wrote the manuscript.

\section{Acknowledgements}

All supports received from Shahid Beheshti University of Medical Sciences (Tehran, Iran) are acknowledged.

\section{References}

1. von Neubeck, C., et al., Glioblastoma multiforme: emerging treatments and stratification markers beyond new drugs. Br J Radiol, 2015. 88(1053): p. 20150354.

2. Villa, S., C. Balana, and S. Comas, Radiation and concomitant chemotherapy for patients with glioblastoma multiforme. Chin J Cancer, 2014. 33(1): p. 25-31.

3. Chalmers, A.J., Overcoming resistance of glioblastoma to conventional cytotoxic therapies by the addition of PARP inhibitors. Anticancer Agents Med Chem, 2010. 10(7): p. 520-33.

4. Hall, S., et al., Protection against Radiotherapy-Induced Toxicity. Antioxidants (Basel), 2016. 5(3).

5. Dombrowsky, A.C., et al., Acute Skin Damage and Late Radiation-Induced Fibrosis and Inflammation in Murine Ears after High-Dose Irradiation. Cancers (Basel), 2019. 11(5).

6. Reisz, J.A., et al., Effects of ionizing radiation on biological molecules--mechanisms of damage and emerging methods of detection. Antioxid Redox Signal, 2014. 21(2): p. 26092.

7. Saleh-Gohari, N., et al., Spontaneous homologous recombination is induced by collapsed replication forks that are caused by endogenous DNA single-strand breaks. Mol Cell Biol, 2005. 25(16): p. 7158-69.

8. Morales, J., et al., Review of poly (ADP-ribose) polymerase (PARP) mechanisms of action and rationale for targeting in cancer and other diseases. Crit Rev Eukaryot Gene Expr, 2014. 24(1): p. 15-28.

9. Penning, T.D., et al., Optimization of phenyl-substituted benzimidazole carboxamide poly(ADP-ribose) polymerase inhibitors: identification of $(S)-2-(2-f l u o r o-4-(p y r r o l i d i n-2-$ 
yl)phenyl)-1H-benzimidazole-4-carboxamide (A-966492), a highly potent and efficacious inhibitor. J Med Chem, 2010. 53(8): p. 3142-53.

10. Koosha, F., et al., The combination of A-966492 and Topotecan for effective radiosensitization on glioblastoma spheroids. Biochem Biophys Res Commun, 2017. 491(4): p. 1092-1097.

11. Tomicic, M.T., M. Christmann, and B. Kaina, Topotecan triggers apoptosis in p53deficient cells by forcing degradation of XIAP and survivin thereby activating caspase-3mediated Bid cleavage. J Pharmacol Exp Ther, 2010. 332(1): p. 316-25.

12. Ersahin, D., I. Doddamane, and D. Cheng, Targeted Radionuclide Therapy. Cancers, 2011. 3(4): p. 3838-3855.

13. Neshasteh-Riz, A., et al., Comparison of DSB effects of the beta particles of iodine-131 and $6 M V X$-ray at a dose of 2 Gy in the presence of 2-Methoxyestradiol, IUdR, and TPT in glioblastoma spheroids. Radiation Physics and Chemistry, 2017. 131: p. 41-45.

14. Mamelak, A.N., et al., Phase I single-dose study of intracavitary-administered iodine-131TM-601 in adults with recurrent high-grade glioma. J Clin Oncol, 2006. 24(22): p. 36443650.

15. Araki, R., et al., Feasibility of high-dose iodine-131-metaiodobenzylguanidine therapy for high-risk neuroblastoma preceding myeloablative chemotherapy and hematopoietic stem cell transplantation: a study protocol. Asia Oceania Journal of Nuclear Medicine and Biology, 2018. 6(2): p. 161.

16. Neshasteh-Riz, A., A.M. Pashazadeh, and S.R. Mahdavi, Relative Biological Effectiveness (RBE) of 1311 Radiation Relative to 60Co Gamma Rays. Cell Journal (Yakhteh), 2013. 15(3): p. 224.

17. Neshasteh-Riz, A., et al., DNA damage induced in glioblastoma cells by I-131: a comparison between experimental data and Monte Carlo simulation. Cell Journal (Yakhteh), 2012. 14(1): p. 25.

18. Gudkov, S.V., et al., Targeted radionuclide therapy of human tumors. International journal of molecular sciences, 2016. 17(1): p. 33.

19. Blasiak, J., DNA-damaging anticancer drugs-A Perspective for DNA repair-oriented therapy. Current medicinal chemistry, 2017. 24(15): p. 1488-1503.

20. Koosha, F., et al., The combination of A-966492 and Topotecan for effective radiosensitization on glioblastoma spheroids. Biochemical and biophysical research communications, 2017. 491(4): p. 1092-1097.

21. Chrisoulidou, A., et al., The diagnosis and management of malignant phaeochromocytoma and paraganglioma. Endocrine-related cancer, 2007. 14(3): p. 569-585.

22. Gedik, G.K., et al., 131 I-MIBG therapy in metastatic phaeochromocytoma and paraganglioma. European journal of nuclear medicine and molecular imaging, 2008. 35(4): p. 725-733.

23. Castellani, M.R., et al., MIBG for diagnosis and therapy of medullay thyroid carcinoma: is there still a role? The Quarterly Journal of Nuclear Medicine and Molecular Imaging, 2008. 52(4): p. 430.

24. Safford, S.D., et al., Iodine-131 metaiodobenzylguanidine treatment for metastatic carcinoid: Results in 98 patients. Cancer, 2004. 101(9): p. 1987-1993.

25. Taal, B.G., et al., Palliative effect of metaiodobenzylguanidine in metastatic carcinoid tumors. Journal of Clinical Oncology, 1996. 14(6): p. 1829-1838. 
26. $\mathrm{Ni}, \mathrm{Y}$., Oncocidia: a small molecule dual targeting pan-anticancer theragnostic strategy. 2014, AACR.

27. Unak, P. and B. Cetinkaya, Absorbed dose estimates at the cellular level for 131I. Applied radiation and isotopes, 2005. 62(6): p. 861-869.

\section{Tables}

Table1. The radiobiological parameters of experimental groups. The average values of $\alpha, \beta$, and SER50 of U87MG cells were estimated by fitting the cell survival to the LQ model.

\begin{tabular}{cccc}
\hline Treatment & $\alpha \pm$ SD & $\beta \pm$ SD & SER $_{50}$ \\
\hline Iodine-131 & $0.00 \pm 0.004$ & $0.04 \pm 0.001$ & - \\
\hline Iodine-131 + TPT & $0.00 \pm 0.02$ & $0.06 \pm 0.01$ & 1.25 \\
\hline $\begin{array}{c}\text { Iodine-131+ A-966492 } \\
\text { Iodine-131 + A-966492 + } \\
\text { TPT }\end{array}$ & $0.02 \pm 0.04$ & $0.07 \pm 0.01$ & 1.45 \\
\hline
\end{tabular}

\section{Figures}



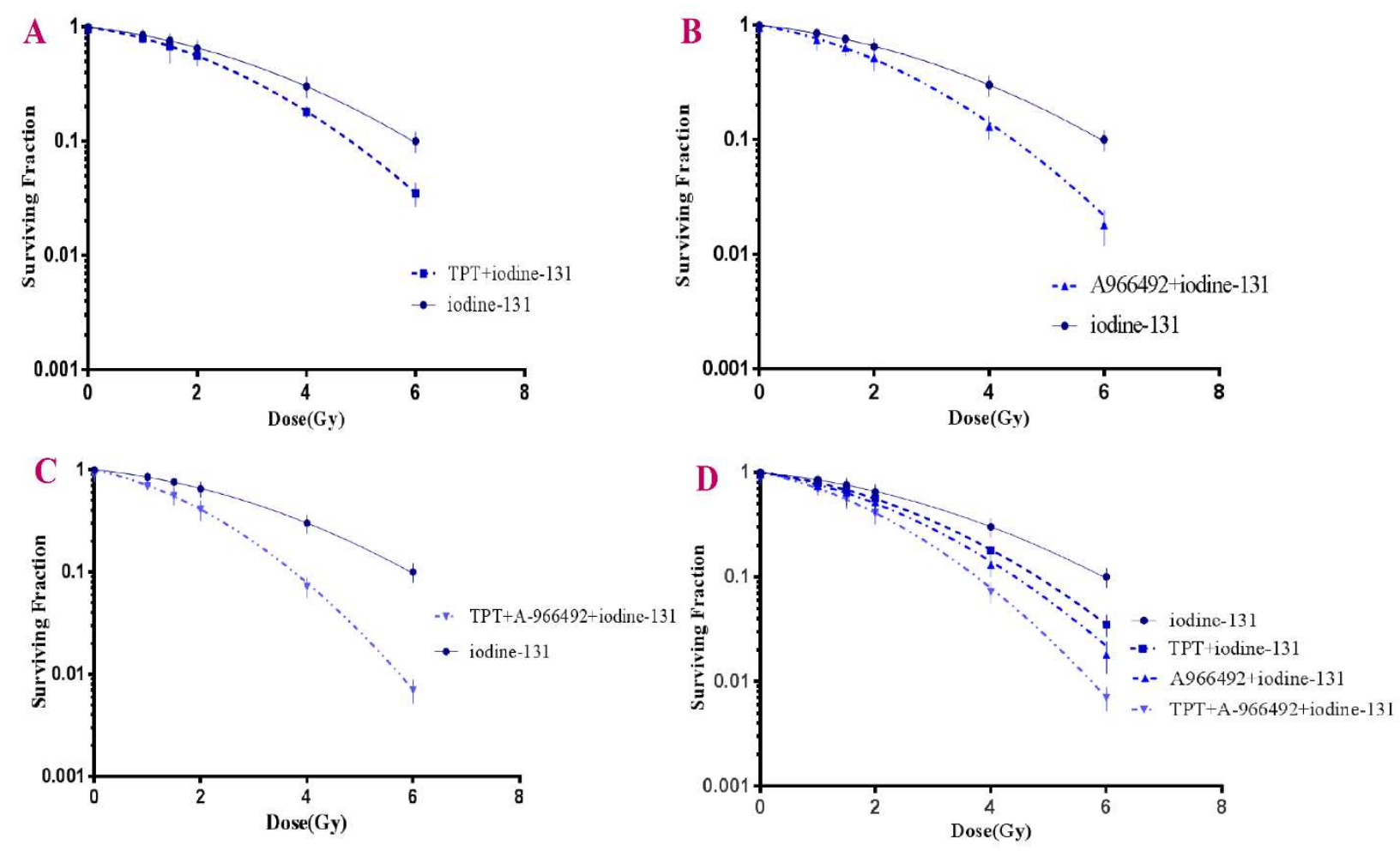

Figure 1. The iodine-131 curves of cell survival in response to treatment with A-966492, TPT, and combination of both. The spheroids were treated with TPT and/or $1 \mu \mathrm{M}$ A-966492 and then irradiated with iodine-131.

The surviving fraction versus dose (Gy); The spheroids were treated with (A) iodine-131 and TPT + iodine-131, (B) iodine-131, and A-966492 iodine-131, (C) and a combination of TPT and A-966492 + iodine-131 (D). The statistical comparison of the obtained data between the experimental groups (A, $\mathrm{B}, \mathrm{C})$; Values are expressed as mean $\pm \mathrm{SD}$ of three independent experiments. 

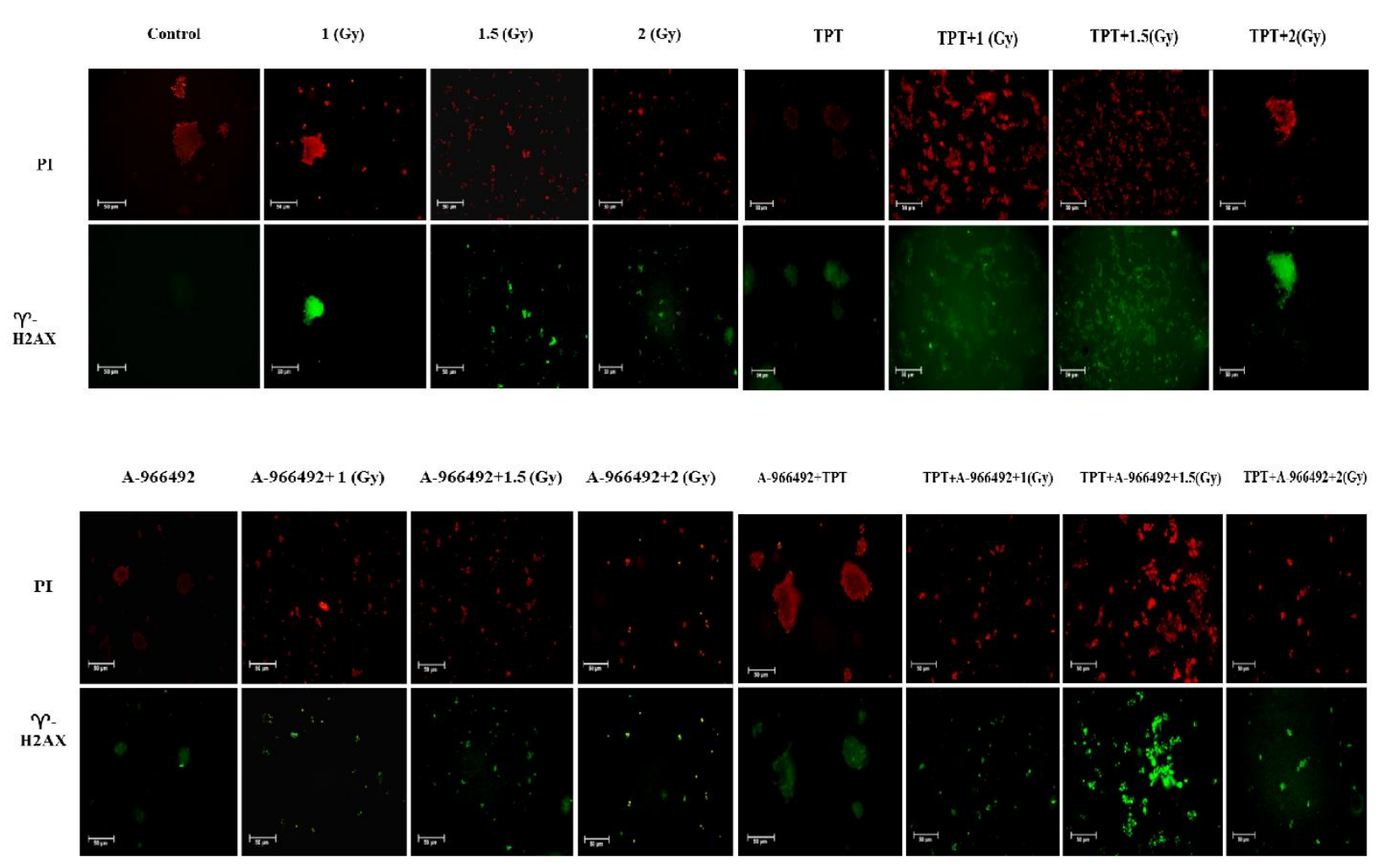

Figure 2. The expression of $\boldsymbol{r}-\mathrm{H} 2 \mathrm{AX}$ shown by immunofluorescence staining.

Cells were treated with $1 \mu \mathrm{M}$ of TPT and/or $1 \mu \mathrm{M}$ of A-966492, then irradiated with iodine-131. Cells were immediately fixed with paraformaldehyde. Row 1 indicates the nuclei of cells, stained with PI while row 2 shows the expression levels of $r-\mathrm{H} 2 \mathrm{AX}$ in cells. 

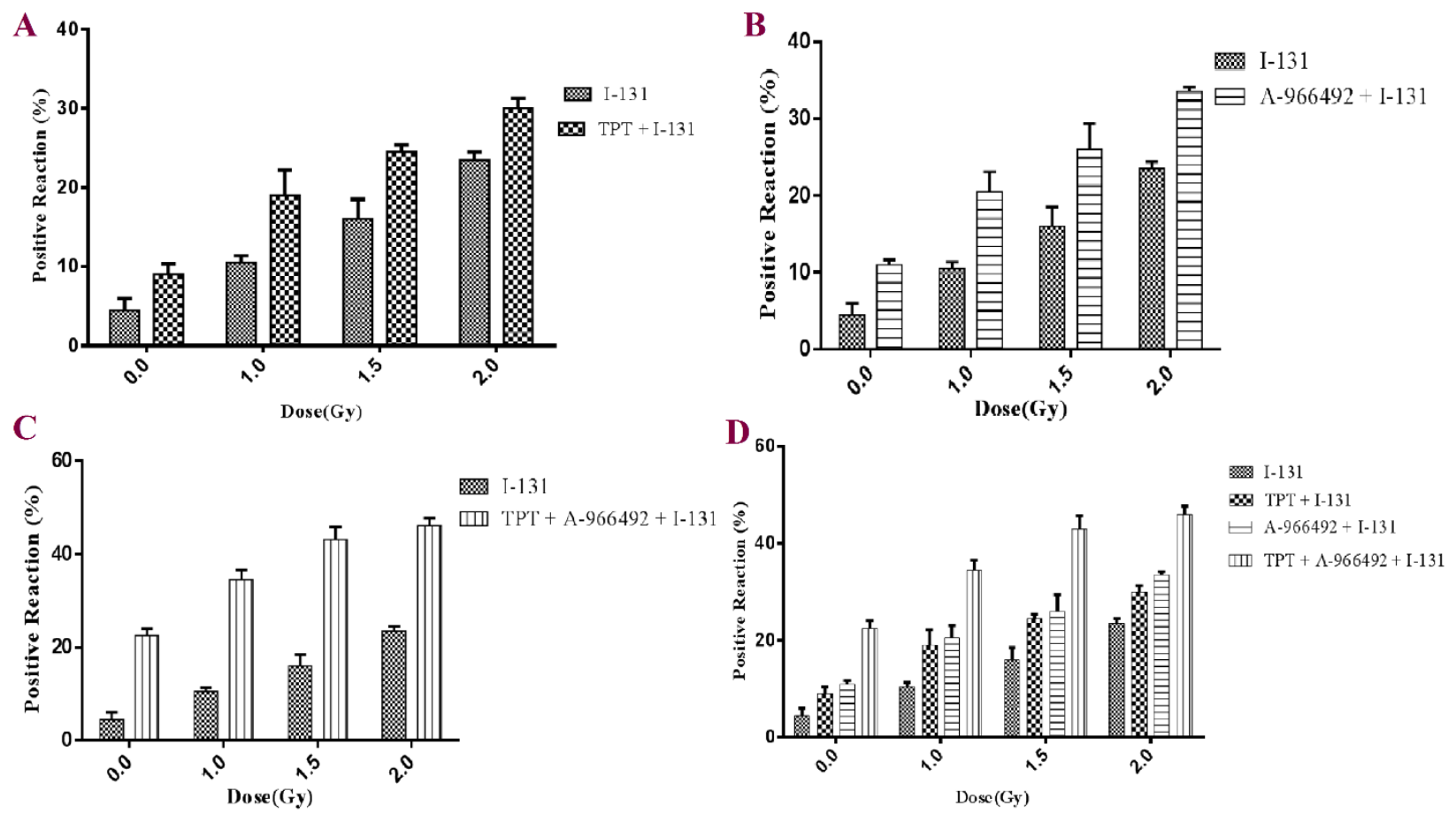

Figure 3. The semi-quantified expression of $\boldsymbol{\gamma}-\mathrm{H} 2 \mathrm{AX}$ analyzed by the ImageJ software. The cells were treated with1 $\mu \mathrm{M}$ TPT and/or $1 \mu \mathrm{M}$ A-966492, then irradiated with iodine-131. Doublestrand break (DSB), categorized as DNA damage, was determined by immunofluorescence staining of phosphorylated histone $\mathrm{H} 2 \mathrm{AX}$ expression. The expression rate of $\gamma-\mathrm{H} 2 \mathrm{AX}$ was plotted versus the radiation dose (Gy). The spheroids were treated with (A) iodine-131 and TPT + iodine131, (B) iodine-131 and A-966492 + iodine-131, (C) a combination of TPT, A-9664 and iodine131, (D) the comparison of the obtained data between the experimental groups (A, B, C). The values are demonstrated as means \pm SD of at least three independent experiments. 
A

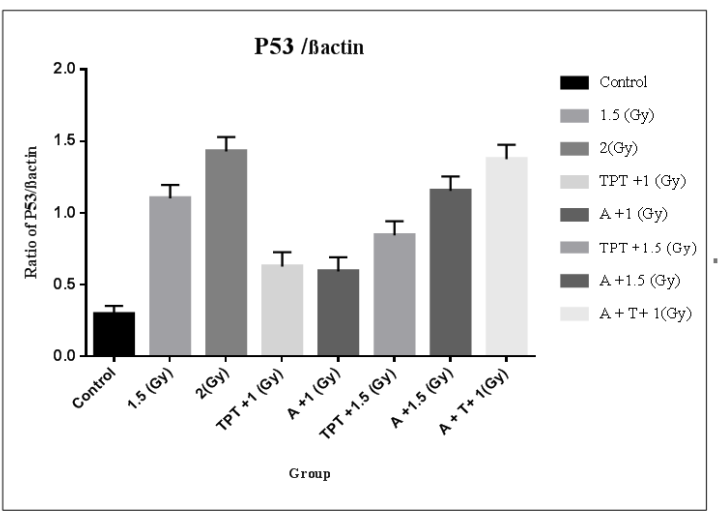

B

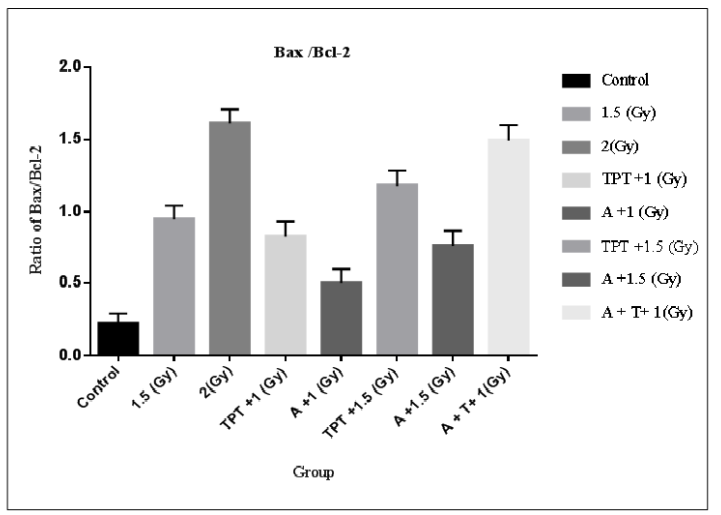

$\mathbf{C}$

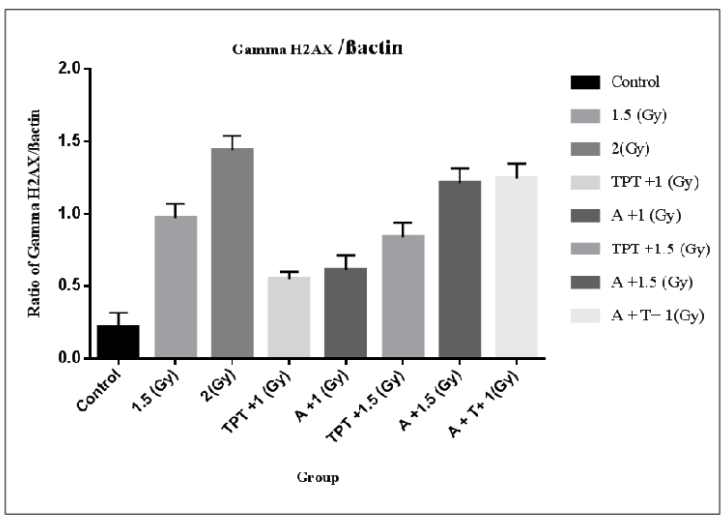

D

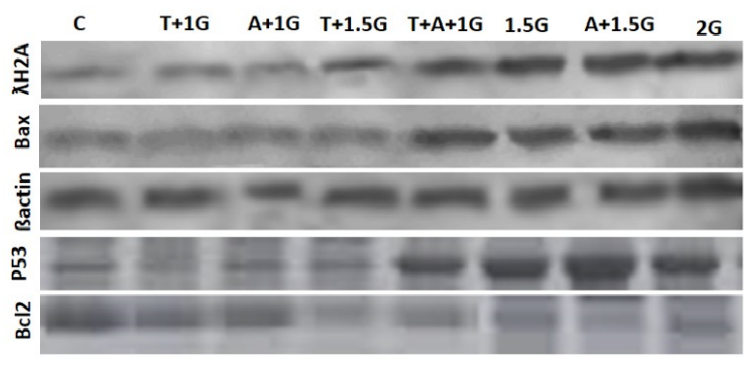

Figure 4. The expression levels of Bax, Bcl-2, p53, and $\mathrm{\gamma}-\mathrm{H} 2 \mathrm{AX}$ were determined by the western blot analysis.

The cells were treated with $1 \mu \mathrm{M}$ of TPT and/or $1 \mu \mathrm{M}$ of A-966492, then irradiated with iodine131. The impact of multiple treatments on expression levels of (A) p53, (B) the ratio of Bax/Bcl2, and (C) Gamma H2AX was assessed by western blot and the obtained values were analyzed by ImageJ software. The beta actin protein was utilized to normalize the data and the assessment of relative expression of each sample. (D) The expression rates of Bax, Bcl-2, p53, $\gamma-\mathrm{H} 2 \mathrm{AX}$, and beta actin were determined by western blot. The above blots are representative of three independent experiments. 
Figures
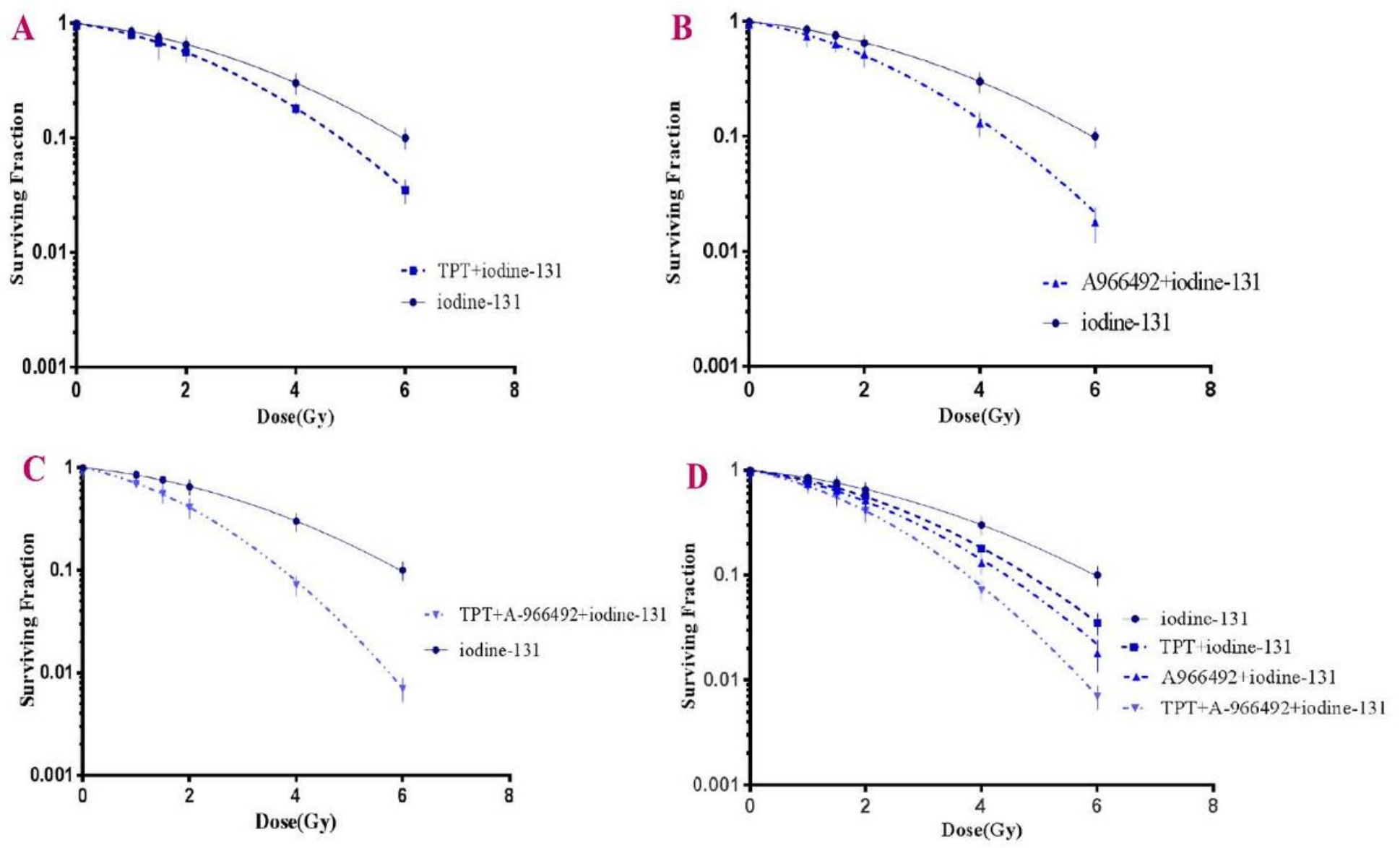

Figure 1

The iodine-131 curves of cell survival in response to treatment with A-966492, TPT, and combination of both . The spheroids were treated with TPT and/or $1 \mu \mathrm{M} \mathrm{A-966492}$ and then irradiated with iodine-131. The surviving fraction versus dose (Gy); The spheroids were treated with (A) iodine-131 and TPT + iodine131, (B) iodine-131, and A-966492 iodine-131, (C) and a combination of TPT and A-966492 + iodine-131 (D). The statistical comparison of the obtained data between the experimental groups $(A, B, C)$; Values are expressed as mean \pm SD of three independent experiments. 


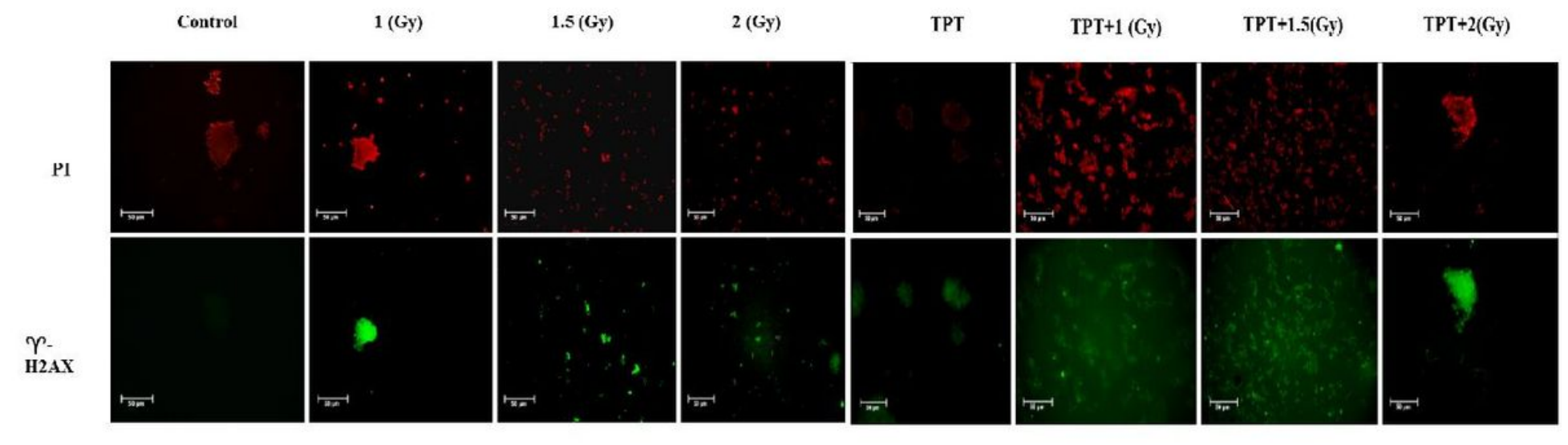

A-966492+TPT

TPT + A- $966492+1(\mathrm{Cry}) \quad$ TPT $+\mathrm{A}-966492+1.5(\mathrm{~Gy})$

$\mathrm{IPT}+\Lambda$ 966492+2(Gy)

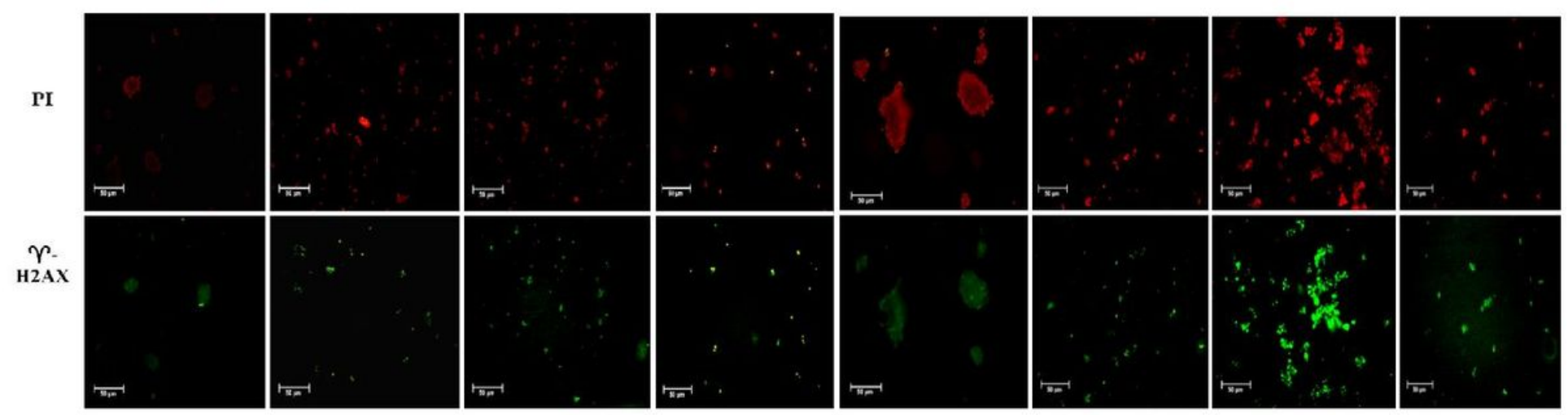

Figure 2

The expression of $\mathrm{y}-\mathrm{H} 2 \mathrm{AX}$ shown by immunofluorescence staining. Cells were treated with $1 \mu \mathrm{M}$ of TPT and/or $1 \mu \mathrm{M}$ of A-966492, then irradiated with iodine-131. Cells were immediately fixed with paraformaldehyde. Row 1 indicates the nuclei of cells, stained with PI while row 2 shows the expression levels of $\mathrm{Y}-\mathrm{H} 2 \mathrm{AX}$ in cells. 

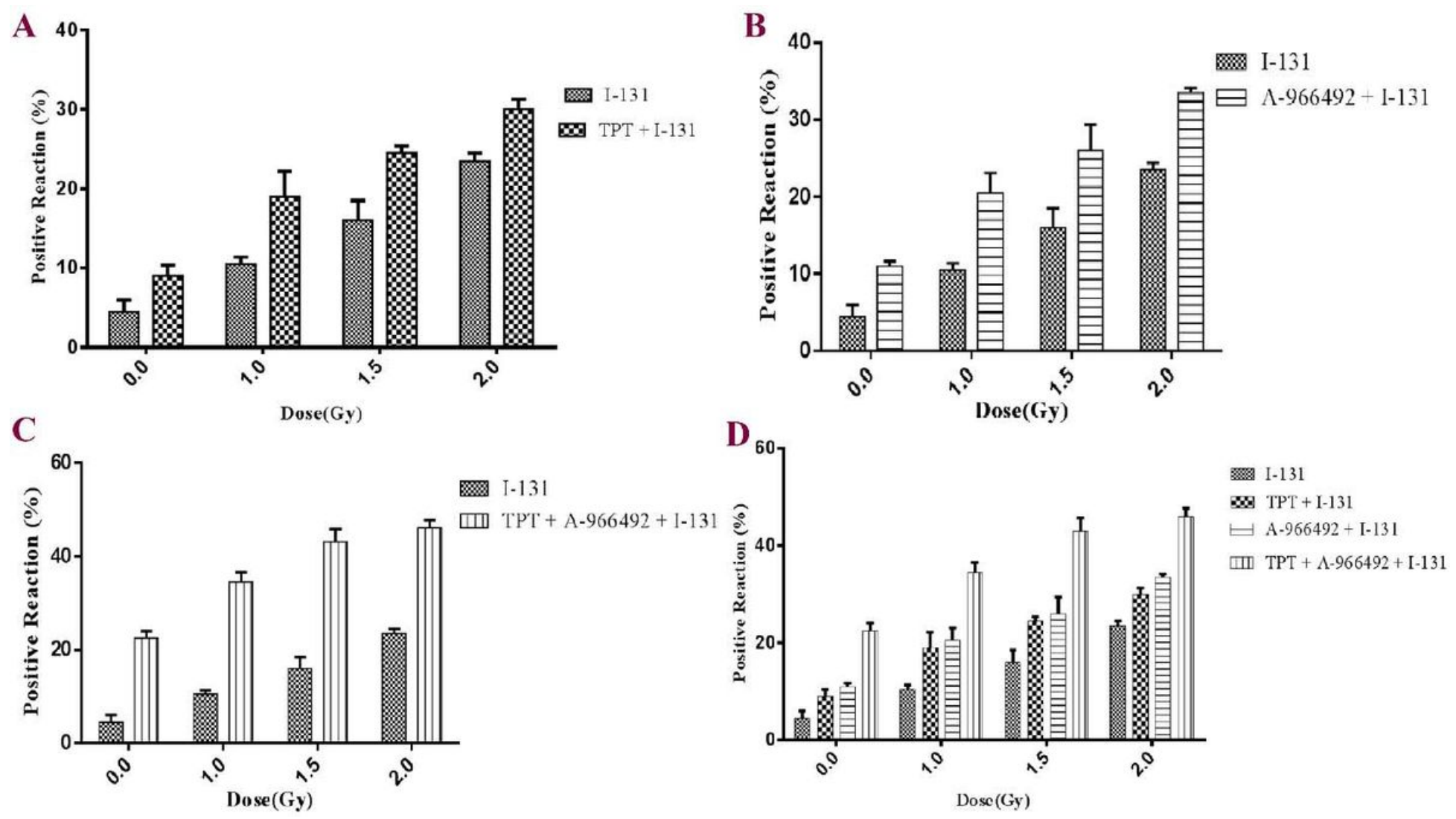

Figure 3

The semi-quantified expression of $\mathrm{Y}-\mathrm{H} 2 \mathrm{AX}$ analyzed by the Image J software. The cells were treated with1 $\mu \mathrm{M}$ TPT and/or $1 \mu \mathrm{M}$ A-966492, then irradiated with iodine-131. Double-strand break (DSB), categorized as DNA damage, was determined by immunofluorescence staining of phosphorylated histone H2AX expression. The expression rate of $\mathrm{Y}-\mathrm{H} 2 \mathrm{AX}$ was plotted versus the radiation dose (Gy). The spheroids were treated with (A) iodine-131 and TPT + iodine-131, (B) iodine-131 and A-966492 + iodine-131, (C) a combination of TPT, A-9664 and iodine-131, (D) the comparison of the obtained data between the experimental groups $(A, B, C)$. The values are demonstrated as means $\pm S D$ of at least three independent experiments. 
$\mathbf{A}$

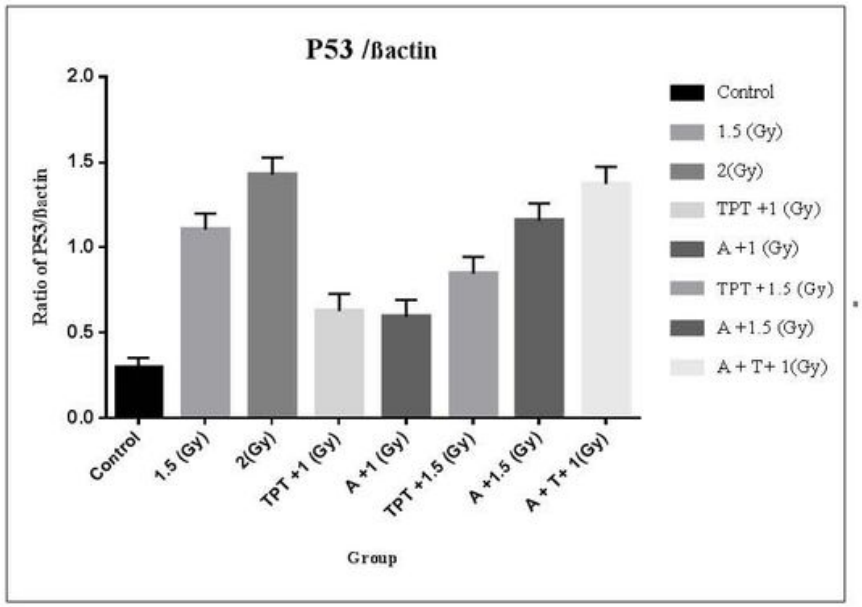

B

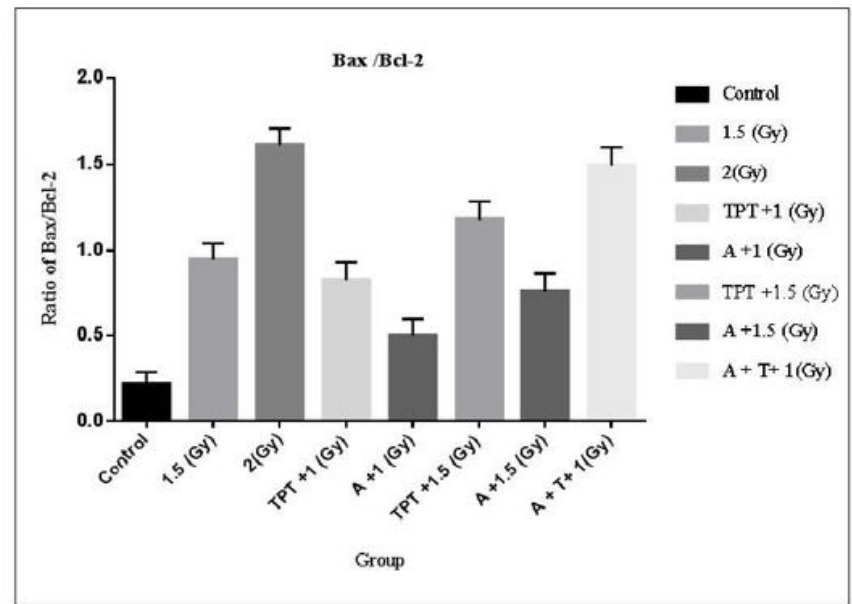

C

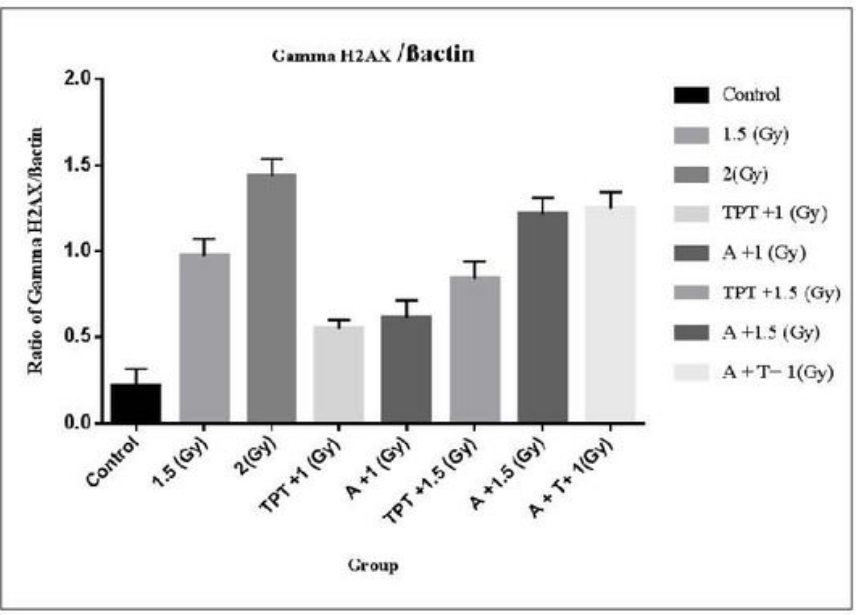

D

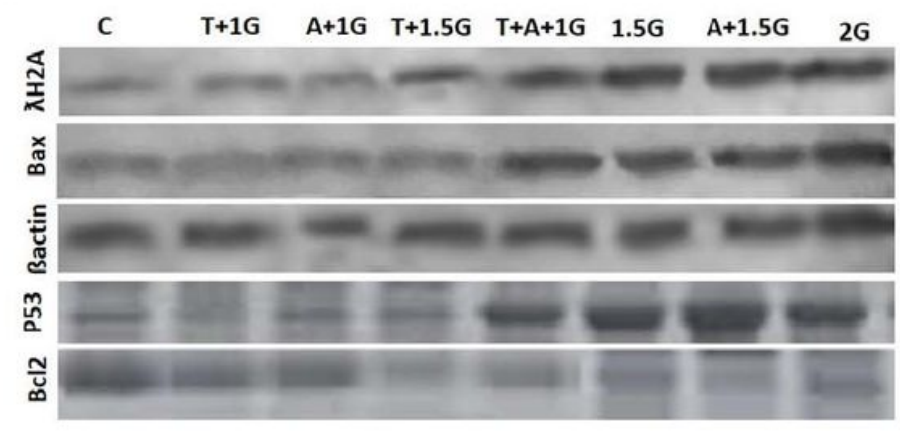

\section{Figure 4}

The expression levels of $\mathrm{Bax}, \mathrm{Bcl}-2, \mathrm{p} 53$, and $\mathrm{y}-\mathrm{H} 2 \mathrm{AX}$ were determined by the western blot analysis. The cells were treated with $1 \mu \mathrm{M}$ of TPT and/or $1 \mu \mathrm{M}$ of $\mathrm{A}-966492$, then irradiated with iodine-131. The impact of multiple treatments on expression levels of (A) p53, (B) the ratio of Bax/Bcl-2, and (C) Gamma H2AX was assessed by western blot and the obtained values were analyzed by ImageJ software. The beta actin protein was utilized to normalize the data and the assessment of relative expression of each sample. (D) The expression rates of $\mathrm{Bax}, \mathrm{Bcl}-2, \mathrm{p} 53, \mathrm{y}-\mathrm{H} 2 \mathrm{AX}$, and beta actin were determined by western blot. The above blots are representative of three independent experiments. 